\title{
Parsonnet score is a good predictor of the duration of intensive care unit stay following cardiac surgery
}

\author{
D R Lawrence, O Valencia, E E J Smith, A Murday, T Treasure
}

\begin{abstract}
Objective-To investigate the value of the Parsonnet score (PS) in identifying preoperatively patients that are likely to spend $<24$ hours on the intensive care unit (ICU) following cardiac surgery.

Method-Prospectively collected data on 5591 patients were analysed. PS, mortality, the length of stay on the ICU (ICU-LOS), number of patients with clinical evidence of stroke, need for haemofiltration, resternotomy for bleeding, tracheostomy, and use of intra-aortic balloon pump were documented as outcomes. A receiver operating characteristic (ROC) curve constructed using PS as a predictor of ICU stay $<24$ hours identified a PS of 10 as the best cut off point that would predict ICU-LOS $<24$ hours. The patients were therefore stratified by PS into two groups, those with a PS of 0 to 9 (PS 0-9) and those with a PS of 10 and above (PS 10+).

Results-The ROC curve constructed using PS as a predictor of ICU stay $<24$ hours had an area under the curve of $0.70(0.01)$. The maximum efficiency of the test was at a sensitivity of 0.68. This corresponded to PS 10. The positive predictive value of the test at this score was $90.5 \%$. Patients with PS $0-9$ had a mean ICU stay of 1.49 days, while patients with PS $10+$ had a mean ICU stay of 2.89 days $(p=0.01)$. The risk of stroke, use of intra-aortic balloon pump, requirement for haemofiltration, need for tracheostomy, and risk of resternotomy for bleeding were each significantly less in patients with PS $0-9$ versus those with a score of PS $10+(p<0.01$ in all cases). The risk of a single complication was 4.7\% (PS 0-9) v15.2\% (PS 10+) (p<0.01). Conclusion-PS is an impartial and objective method of predicting postoperative complications and ICU stay $<24$ hours. This is of value in selecting a cohort of patients likely to maintain a smooth flow of patients through the cardiothoracic unit when resources are limited to a few free ICU beds.
\end{abstract}

(Heart 2000;83:429-432)

Keywords: Parsonnet score; intensive care; length of hospital stay; rationing

In a climate of limited intensive care resources, clinicians are often required to identify patients that are likely to require a short period of high dependency care following cardiac surgery. The number of available intensive care unit (ICU) beds may be limited by a high number of long stay patients or more commonly because of a shortage of trained cardiac ICU nurses. One method of maximising the throughput of the unit under these circumstances is to operate selectively on patients that are likely to require $<24$ hours of ICU treatment following cardiac surgery.

The identification of such cases can be controversial. Experienced clinicians have been shown to be able to predict ICU length of stay (ICU-LOS) in days in just $51 \%$ of patients. ${ }^{1}$ They were, however, able to identify patients who will spend $<48$ hours on the ICU on $87 \%$ of occasions. The operating consultant surgeon must use theatre time efficiently and is mindful of the ever increasing waiting list. Consequently, interpretation of a patient's likelihood of requiring $<24$ hours ICU care may differ considerably from that of the surgeon due to operate the following day whose list will be cancelled if the said patient were to require 48 hours of ICU care. We have assessed the value of the Parsonnet score (PS), which has the advantages of being a relatively non-negotiable score that cardiologists, cardiac surgeons, and anaesthetists are familiar with, as a predictor of ICU stay $<24$ hours after cardiac surgery.

\section{Patients and methods}

We examined the records of 5591 patients that underwent cardiac surgery in our unit. We excluded patients that died in the operating theatre and those that died within 24 hours of transfer to the ICU $(n=42)$. The following outcomes were recorded: duration of stay on the ICU in days; number of patients with

Table 1 Distribution of PS and mortality $(n=5591)$

\begin{tabular}{llllll}
\hline \multicolumn{5}{l}{ Parsonnet score } \\
& $0-4$ & $5-9$ & $10-14$ & $15-19$ & $>20$ \\
\hline $\mathrm{n}$ & 2189 & 1363 & 845 & 526 & 66 \\
Died (\%) & $39(1.8)$ & $54(3.9)$ & $64(7.6)$ & $50(9.5)$ & $127(19)$ \\
\hline
\end{tabular}

Table 2 Postoperative ICU-LOS for those patients with PS of 0 to 9, and those with PS of 10 and above

\begin{tabular}{llll}
\hline & PS 0-9 & PS 10+ & p Value \\
\hline Mean ICU stay (days) & 1.49 & 2.89 & $<0.01$ \\
$\begin{array}{l}\text { Median ICU stay (days) } \\
\text { IQR (days) }\end{array}$ & $1-1$ & $1-2$ & $<0.01$ \\
$\begin{array}{l}\text { 10th centile-90th centile } \\
\text { (days) }\end{array}$ & $1-1$ & $1-5$ & $<0.01$ \\
\hline
\end{tabular}

IQR, interquartile range. 


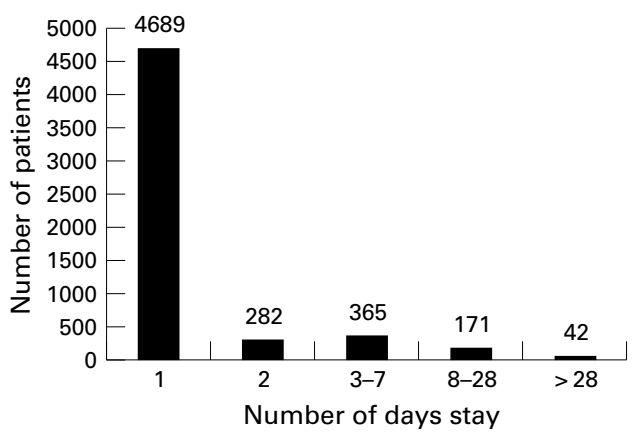

Figure 1 ICU-LOS versus number of patients $(n=5549)$.

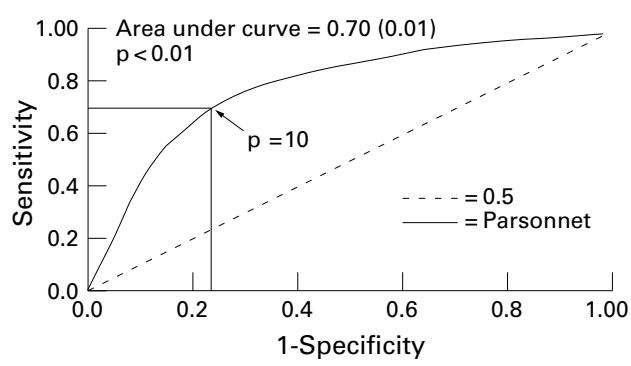

Figure 2 ROC for PS as a predictor of ICU-LOS $<24$ hours. Sensitivity of 0.68 and specificity of 0.77 when PS is 10.

clinical evidence of stroke; need for haemofiltration; resternotomy for bleeding; tracheostomy; and use of intra-aortic balloon pump. The in-hospital mortality was also documented.

A receiver operating characteristic curve (ROC) was constructed for evaluation of PS as a predictor of postoperative ICU-LOS of $<24$ hours. This was performed by using each PS (from 1 to 60 ) as a theoretical cut off point to predict ICU-LOS of $<24$ hours. The sensitivity and specificity of the prediction were calculated for each PS, based on the observed duration of ICU stay. We then plotted sensitivity versus 1 -specificity. The maximum efficiency of the test was identified as the point at which the rate of change of the tangent to the curve is maximal-that is, for a small increase in sensitivity the compromise in specificity becomes large. A horizontal line plotted back to the y axis then identified the sensitivity at which the test was most efficient (sensitivity $=0.68$ ). The value of the PS that produced this sensitivity was then identified from the original data sheet (PS $=10)$.

Having identified a PS of 10 as the most valuable score, the patients were stratified into two groups: those with a PS of 0 to 9 (PS 0-9) ( $\mathrm{n}=3546)$; and those with a PS of 10 and above (PS 10+) $(n=2003)$. Results were ana-

Table 3 Data for PS 8 to 12. Sensitivity and specificity are for predicting ICU-LOS $<24$ hours

\begin{tabular}{rlll}
\hline PS & Sensitivity & Specificity & $\begin{array}{l}\text { Sample that qualify } \\
\text { for operation (\%) }\end{array}$ \\
\hline 8 & 0.62 & 0.80 & 56.6 \\
9 & 0.65 & 0.78 & 59.8 \\
10 & 0.68 & 0.77 & 63.4 \\
11 & 0.73 & 0.70 & 67.5 \\
12 & 0.75 & 0.65 & 70.4 \\
\hline
\end{tabular}

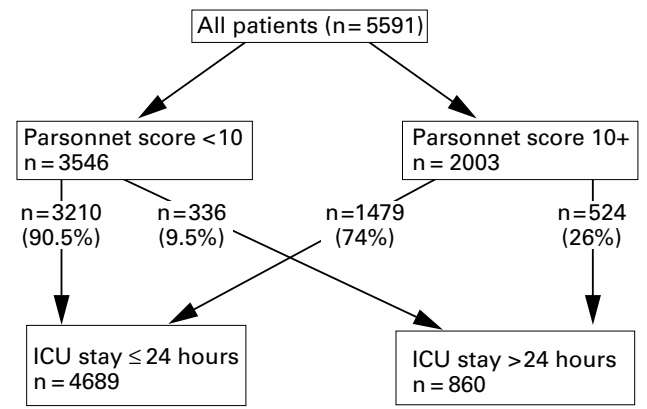

Figure 3 Flow chart of patients passing through ICU using PS as a sieve.

lysed using a $\chi^{2}$ test for comparing outcomes between the two groups and a Mann Whitney $\mathrm{U}$ test for comparing distribution of ICU-LOS.

\section{Results}

CASE DISTRIBUTION

The 5591 patients included 3996 (71.5\%) cases of coronary artery bypass surgery and 825 cases $(14.8 \%)$ of single valve replacement, as well as 42 patients who died at operation or within 24 hours of transfer to the ICU. The overall distribution of PS and mortality are shown in table 1 .

DURATION OF ICU STAY

The duration of ICU stay is shown in table 2, and the distribution of ICU-LOS is shown in fig 1.

EFFICIENCY IN PREDICTING ICU-LOS $<24$ HOURS ROC analysis was used to assess how efficiently PS predicted an ICU-LOS of $<24$ hours (fig 2). The area under the curve was 0.7 (0.01) units. The data for PS 8 to 12 are shown in table 3. A PS of 9 generated a sensitivity of 0.65 with a specificity of 0.78 . By using a PS of 10 as a cut off value the test achieved a sensitivity of 0.68 (a gain of 3 points) with a specificity of 0.77 (a loss of 1 point versus a PS of 9). Using a PS of 11 results in a sensitivity of 0.73 (a further gain of 5 points) with a specificity of 0.7 (a loss of 7 points versus a PS of 10). We therefore identified a PS of 10 as the best predictor of ICU-LOS of $<24$ hours. The positive predictive value of the test at this PS was $90.5 \%$ with a negative predictive value of $26.1 \%$ (fig 3).

\section{COMPLICATIONS}

The risk of suffering a clinically detectable stroke, use of intra-aortic balloon pump, use of haemofiltration, requirement of resternotomy for bleeding, and need for tracheostomy are shown in table 4.

Table 4 Risks of complication by PS $(n=5549)$

\begin{tabular}{lcrl}
\hline & PS 0-9 & PS 10+ & p Value \\
\hline Cerebrovascular accident & & & \\
$\quad$ (stroke) & $1.0 \%$ & $3.5 \%$ & $<0.01$ \\
Intra-aortic balloon pump & $2.7 \%$ & $8.4 \%$ & $<0.01$ \\
Haemofiltration & $2.1 \%$ & $12.3 \%$ & $<0.01$ \\
Resternotomy & $3.4 \%$ & $6.1 \%$ & $<0.01$ \\
Tracheostomy & $0.9 \%$ & $4.2 \%$ & $<0.01$ \\
Risk of at least one & & & $<0.01$ \\
$\quad$ complication & $4.7 \%$ & $15.2 \%$ & $<0.01$ \\
\hline p Value, degree of significance between PS 0-9 versus PS $10+$.
\end{tabular}


Table 5 PS calculator currently used in our unit

\begin{tabular}{lr}
\hline & $P S$ \\
\hline Female sex & 1 \\
Morbid obesity & 3 \\
Diabetes (type unspecified) & 3 \\
Hypertension (systolic blood pressure > $140 \mathrm{~mm} \mathrm{Hg}$ & 3 \\
Left ventricular ejection fraction & \\
$\quad$ Good $(>49 \%)$ & 0 \\
Fair $(30-49 \%)$ & 2 \\
Poor (<30\%) & 4 \\
Age & \\
70-74 years & 7 \\
$75-79$ years & 12 \\
$>79$ years & 20 \\
Reoperation & \\
First & 5 \\
Second & 10 \\
Preoperative intra-aortic balloon pump & 2 \\
Left ventricular aneurysm & 5 \\
Emergency surgery following coronary angioplasty & 10 \\
Dialysis dependency & 10 \\
Valve surgery & \\
Mitral & 5 \\
If pulmonary artery pressure > 60 mm Hg add: & 8 \\
Aortic & \\
If gradient > 120 mm Hg add: & 5 \\
CABG at time of valve surgery & 7 \\
\end{tabular}

Catastrophic state (for example, patient receiving cardiopulmonary resuscitation while being transferred into operating theatre) add score of $10-50$ (very rarely used).

$\mathrm{CABG}$, coronary artery bypass graft.

\section{Discussion}

In a world of limited medical facilities but increasing demand for medical services, rationing is something all members of the medical profession and their patients are familiar with. Much time and effort have been spent trying to identify patients who would benefit from cardiac surgery and attempting to prioritise their position on waiting lists. ${ }^{2}$ It was quickly recognised, however, that decisions about priority and urgency were often inconsistent. ${ }^{2}$ The advantage of having a simple and objective scoring system for establishing surgical priority was promoted by the New Zealand priority criteria project. ${ }^{3}$ In that study four criteria were assessed: degree of coronary artery obstruction; angina status; exercise test result; and ability to work, care for dependents, or live independently. Patients were allocated a score of $0-100$. The authors concluded that patients with a score of 25 would have a better prognosis if they underwent coronary artery surgery. The New Zealand score addresses objectively the issue of priority according to deservedness.

PS, however, ${ }^{4}$ was developed as a tool for predicting the risk of death following adult cardiac surgery (table 5). Improvements in anaesthesia, modern day intensive care, extracorporeal circulation, and myocardial preservation have contributed to lower mortality rates than those predicted by PS. The scoring system has been criticised as it does not take into account the quality of a patient's coronary arteries in predicting operative risk, and probably overestimates the risk of age. There have been several other computerised attempts at predicting operative risk more accurately but this is always at the expense of simplicity and practicality of use. PS has therefore become a familiar and workable tool for cardiologist and cardiac surgeon alike.
In our series of 5549 patients, the mean ICU-LOS for patients with PS 0-9 was significantly less than for those with PS $10+$. In order to assess the value of the score as a predictor of ICU-LOS of up to 24 hours, an ROC curve was constructed. The positive predictive value of $90.5 \%$ indicates that patients with PS of $<10$ will spend $<24$ hours on the ICU on over $90 \%$ of occasions. The negative predictive value of $26 \%$ implies that $74 \%$ of patients with PS of 10 or greater will be unfairly penalised as they will also require $<24$ hours of ICU care. Nevertheless a high positive predictive value is obviously of greater importance than a high negative predictive value as just one long stay patient can substantially affect a unit's throughput.

While accepting that this test does not achieve ROC values of $>90 \%$, which are required for diagnostic tests such as determination of serum creatine kinase concentrations for the diagnosis of acute myocardial infarction, ${ }^{5}$ our curve achieved a respectable value of 0.70 . Tu and colleagues used a six variable risk index (age, sex, left ventricular function, type of surgery, urgency of surgery, and repeat operation) and found it was able to predict with an ROC value of 0.66 an ICU-LOS of $>6$ days. ${ }^{6}$ It may be possible to increase the specificity of the test by using selective criteria from the PS or by adjusting the values assigned to some parameters, but this would no longer then be a score that we are all familiar with.

Predictors of prolonged ICU-LOS and inpatient LOS have been sought in the past. Weintraub and colleagues showed that age, postoperative wound infection, pneumonia, arrhythmias, neurological events, postoperative infarction, and ejection fraction are associated with a prolonged inpatient LOS. ${ }^{7}$ With the exception of age, however, none of these factors are known preoperatively. Hammermeister and colleagues showed that surgical priority, age, peripheral vascular disease, and high serum creatinine contributed to prolonged inpatient LOS. ${ }^{8}$ Mounsey and colleagues, however, found that age, sex, obesity, diabetes, hypertension, obstructive airways disease, and unstable angina were not predictive of ICU-LOS, but left ventricular end diastolic pressure and the number of diseased coronary arteries were. ${ }^{9}$

We therefore looked at how good PS was at predicting some of the postoperative complications that we associate with increased ICULOS. We looked at clinical evidence of stroke, postoperative use of intra-aortic balloon pump, resternotomy for bleeding, tracheostomy, and use of haemofilter. ${ }^{10}$ In all cases there was a significantly lower incidence in patients with PS $<10$ ( $\mathrm{p}<0.01$ in all cases) (table 4).

In conclusion, the increasing burden placed on the health service by an ageing population demanding more sophisticated medical and surgical procedures has meant that rationing has become necessary. When resources in the ICU are sparse it is necessary to identify a group of patients with a high probability of requiring $<24$ hour ICU care in order that 
some operations can be carried out each day. A PS of $<10$ is a good predictor of such patients. It is a simple score and has the added advantage that it is one with which we are all familiar.

1 Tu JV, Mazer CD. Can clinicians predict ICU length of stay following cardiac surgery? Can f Anaesth 1996;43:789-94.

2 Naylor CD, Baigrie RS, Goldman BS, et al. Assessment of priority for coronary revascularisation procedures. Lancet 1990;335:1070-3.

3 Hadorn DC, Holmes AC. The New Zealand priority criteria project. Part 2: coronary artery bypass graft surgery. BMF 1997:314:135-8.

4 Parsonnet V, Dean D, Bernstein AD. A method of uniform stratification of risk for evaluating the results of surgery in acquired adult heart disease. Circulation 1989;79(suppl I):I-3-12.
5 Collinson P. Of bombers, radiologists and cardiologiststime to ROC. Heart 1998;80:217.

6 Tu JV, Jaglal SB, Naylor D, et al. Multicenter validation of a risk index for mortality, intensive care unit stay and overall hospital length of stay after cardiac surgery. Circulation 1995;91:677-84.

7 Weintraub WS, Jones EL, Craver J, et al. Determinants of prolonged length of hospital stay after coronary bypass surgery. Circulation 1989;80:276-84.

8 Hammermeister KE, Burchfiel C, Johnson R, et al. Identification of patients at greatest risk for developing major complications at cardiac surgery. Circulation 1990;82 (suppl IV):IV-380-9.

9 Mounsey JP, Griffith MJ, Heaviside DW, et al. Determinants of the length of stay in intensive care and in hospital after coronary artery surgery. Br Heart 7 1995;73:92-8.

10 Unsworth-White MJ, Herrriot A, Valencia O, et al. Resternotomy for bleeding after cardiac operation: a marker for increased morbidity and mortality. Ann Thorac Surg 1995;59:664-7.

\section{IMAGES IN CARDIOLOGY}

\section{Postcoarctation giant aneurysm of aorta}

A 16 year old girl came to our department for evaluation of severe hypertension. She was asymptomatic but clinical examination showed signs suggestive of coarctation of aorta. ECG showed left ventricular hypertrophy with strain pattern. Chest radiography showed normal cardiothoracic ratio, prominent smooth bulge in left upper cardiac silhouette, and bilateral rib notching. Echocardiography revealed concentric left ventricular hypertrophy and coarctation segment just after the origin of left subclavian artery. However, the aorta distal to the coarctation segment was not well visualised.

Doppler echocardiography detected peak gradients of $78 \mathrm{~mm} \mathrm{Hg}$ across the coarctation segment. On cardiac catheterisation pull back aortic gradients of $80 \mathrm{~mm} \mathrm{Hg}$ were recorded. Angiocardiography showed severe coarctation of the aorta just distal to the origin of the left subclavian artery and a huge postcoarctation aortic aneurysm (open arrow) with calcification in its wall (closed arrow). Findings were confirmed during surgery and the patient was treated successfully by resection of coarctation segment as well as postcoarctation aneurysm with graft tube interposition. She made an uneventful recovery.

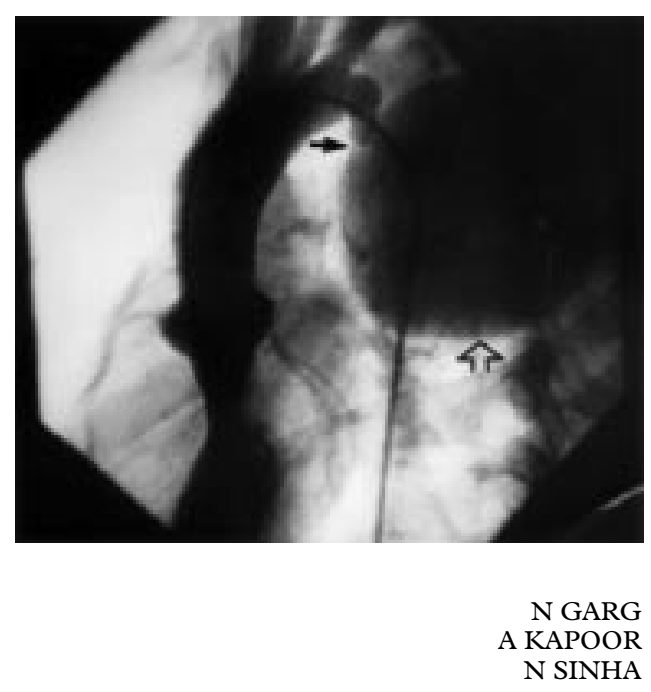

